\title{
Crystal texture of mineral self-organized structures from soda lake water and their implication to early Earth and prebiotic chemistry
}

\author{
M. Getenet, J.M. García-Ruiz \\ Laboratorio de Estudios Cristalográficos, Instituto Andaluz de Ciencias de la Tierra, Consejo Superior de Investigaciones \\ Cientificas-Universidad de Granada, Granada, Spain \\ juanmanuel.garcia@csic.es
}

The ability of minerals to precipitate into complex shapes and textures creates fascinating patterns that has not been enough explored in natural scenarios. Among them, silica induced mineral self-organized structures have been suggested to be relevant for the earliest stages of the planet, when alkaline silica-rich oceans evolve from methane-rich to $\mathrm{CO}$ and $\mathrm{CO}_{2}$-rich atmosphere and hydrosphere [1]. Under these geochemical conditions of the Hadean Earth, it is thought that silica-carbonate biomorphs and silica-metal hydr(oxide) gardens were actually forming in the alkaline oceans, rich in silica and in carbonate. In this work, we focus on chemical gardens, which are hollow membranes formed via abiotic precipitation when metal salts immerse into aqueous solutions containing anions such as silicate, carbonate, or phosphates [2]. It has been shown that these space-compartmentalized membranes are small batteries [3] that selectively catalyse the synthesis of prebiotically relevant compounds such as carboxylic acids, amino acids, and nucleobases by condensation of formamide [4]. Here, we experimentally demonstrate the formation of carbonate gardens using carbonate-rich alkaline soda lake water (Lake Magadi, Southern Kenyan rift valley). We have studied in detail the mineral composition and crystallinity of these "natural" carbonate gardens by SEM-EDX, Raman microscopy, infrared spectroscopy and X-ray diffraction, and compared to other silica and carbonate gardens made from laboratory solutions. Our result suggests that mineral self-organization could have been a geochemically plausible phenomenon in carbonate-rich closed basin environments of the early Earth, and Earth-like planets. We also discuss the implications of the textural properties of the mineral membranes to develop electrochemical potential that could catalyze prebiotic reactions.

[1] García-Ruiz, J.M., van Zuilen, M. \& Bach, W. (2020). Phys Life Rev. 34-35, 62-82.

[2] Kellermeier, M., Glaab, F., Melero-García, E., \& García-Ruiz, J. M. (2013). Research Methods in Biomineralization Science, edited by J.J. De Yoreo, pp. 225-256. San Diego: Academic Press.

[3] Glaab, F., Kellermeier, M., Kunz, W., Morallon, E. \& Garcia-Ruiz, J. M. (2012). Angew. Chem. 124, 4393.

[4] Saladino, R., Di Mauro, E. \& García-Ruiz, J. M. (2019). Chem. Eur. J. 25, 3181.

Keywords: chemical gardens; self-organization; biomorphs; early Earth; Soda lakes

Acknowledgments: We acknowledge funding from the European Research Council under grant agreement no. 340863, from the Ministerio de Economia y Competitividad of Spain through the project CGL2016-78971-P and Junta de Andalucia for financing the project P18-FR-5008. M.G. acknowledges Grant No. BES-2017-081105 of the Ministerio de Ciencia, Innovacion y Universidades of the Spanish government. 